\title{
A Fourier error analysis for radial basis functions and the Discrete Singular Convolution on an infinite uniform grid, Part 1: Error theorem and diffusion in Fourier space
}

\author{
John P. Boyd* \\ Department of Atmospheric, Oceanic and Space Science, \\ University of Michigan, 2455 Hayward Avenue, Ann Arbor MI 48109 \\ jpboyd@umich.edu;
}

March 12, 2015

\begin{abstract}
On an infinite grid with uniform spacing $h$, the cardinal basis $C_{j}(x ; h)$ for many spectral methods consists of translates of a "master cardinal function", $C_{j}(x ; h)=C(x / h-j)$. The cardinal basis satisfies the usual Lagrange cardinal condition, $C_{j}(m h)=\delta_{j m}$ where $\delta_{j m}$ is the Kronecker delta function. All such "shift-invariant subspace" master cardinal functions are of "localized-sinc" form in the sense that $C(X)=\operatorname{sinc}(X) s(X)$ for a localizer function $s$ which is smooth and analytic on the entire real axis and the Whittaker cardinal function is $\operatorname{sinc}(X) \equiv \sin (\pi X) /(\pi X)$. The localized-sinc approximation to a general $f(x)$ is $f^{\text {localized-sinc }}(x ; h) \equiv$ $\sum_{j=-\infty}^{\infty} f(j h) s([x-j h] / h) \operatorname{sinc}([x-j h] / h)$. In contrast to most radial basis function applications, matrix factorization is unnecessary. We prove a general theorem for the Fourier transform of the interpolation error for localized-sinc bases. For exponentially-convergent radial basis functions (RBFs) [Gaussians, inverse multiquadrics, etc.] and the basis functions of the Discrete Singular Convolution (DSC), the localizer function is known exactly or approximately. This allows us to perform additional error analysis for these bases. We show that the error is similar to that for sinc bases except that the localizer acts like a diffusion in Fourier space, smoothing the sinc error.
\end{abstract}

Keywords: radial basis functions; spectral methods; Gaussian radial basis; discrete singular convolution; RBF; DSC interpolation

MSC Subject Classifications: 65D05, 42A15, 43A50 


\section{Introduction}

The sinc pseudospectral method is an exponentially-convergent and easily programmed tool to approximate smooth functions and solve differential equations on an unbounded interval $(x \in \mathbb{R})$ with uniform grid spacing $h[23,35,36]$. The interpolation points (for this basis and all bases throughout this article) are

$$
x_{j}=j h, \quad j=0, \pm 1, \pm 2, \ldots \infty
$$

and the approximation to a function $f(x)$ is

$$
f(x) \approx f^{\operatorname{sinc}}(x) \equiv \sum_{j=-\infty}^{\infty} f(j h) \operatorname{sinc}([x-j h] / h)
$$

where $\operatorname{sinc}(X) \equiv \sin (\pi X) /(\pi X)$.

The sinc basis is a "cardinal" or "Lagrange" basis in the sense that, defining

$$
\begin{gathered}
C_{j}(x ; h) \equiv \operatorname{sinc}(x / h-j) \\
C_{j}\left(x_{i}\right)=\left\{\begin{array}{ll}
1, & i=j \\
0, & i \neq j
\end{array} \text { all integer } i, j\right.
\end{gathered}
$$

The cardinal property implies that the coefficients of the cardinal series are simply the "grid point values" or "samples" of $f(x)$, the set $f\left(x_{j}\right)$.

The sinc basis for a given grid spacing $h$ spans a "shift-invariant subspace" in the sense that all the cardinal functions are translations of a single "master cardinal function" $C(X)$, which is notationally distinguished by the lack of a subscript

$$
C_{j}(x ; h) \equiv C([x-j h] / h)
$$

where $C(X) \equiv \operatorname{sinc}(X)$ for the sinc basis.

Note that the cardinal function for $h \neq 1$ is just the dilation of the cardinal function for unit $h$. When the grid points are uniformly spaced, it is always possible to rescale the spatial coordinate to unit spacing without changing the accuracy of the approximation or the cardinal series coefficients

$$
X=x / h \quad \Leftrightarrow \quad x=h
$$

We shall simplify some formulas by giving them for $h=1$, indicated by using $X$ and $K$ as the spatial coordinate and Fourier Transform argument, respectively. There is no loss of generality because of this change of coordinate.

Although spectrally accurate, the sinc method has the large disadvantage that its differentiation matrices are dense and it is not possible to circumvent dense matrix manipulations by using the Fast Fourier transform, which is inapplicable to the sinc basis. However, a wide variety of other "shift-invariant" bases are available.

The sinc basis is unusual in that it is always written in sinc form. Other spectral bases are usually defined by sets of functions that lack the cardinal 
property. However, it is always possible with any basis set and interpolation point set to take linear combinations $C_{j}$ of the basis functions so that the new basis functions do satisfy (5).

In order to satisfy the cardinal property (for unit grid), the master cardinal function must vanish at all integers except the origin. This implies that all shift-invariant uniform cardinal bases can be written in sinc-factored form as

$$
C(X) \equiv \operatorname{sinc}(X) s(X)
$$

where $s(X)$ is a "localizer" function that is analytic for all real $x$ with $s(0)=1$.

Below, we shall prove a theorem that explicitly gives the error of the interpolating approximation for general $s(X)$. Later, we shall specialize to a couple of particular classes of shift-invariant, uniform grid bases (DSC and RBF, defined below) to analyze the approximation error more precisely.

Guowei Wei and his collaborators have published an extensive series of articles about a modified sinc pseudospectral method that they dubbed the Discrete Singular Convolution (DSC). This is identical to the standard sinc expansion except that the basis functions are localized by the substitution

$$
\operatorname{sinc}(X) \rightarrow s(X) \operatorname{sinc}(X)
$$

where $s(X)$ is a user-chosen localizer function that decays rapidly as $|X| \rightarrow \infty$. Because of the $s(X)$ factor, the modified master cardinal function $C(X) \equiv$ $s(X) \operatorname{sinc}(X)$ now decays rapidly away from its peak at the origin. This makes it possible to truncate the DSC differentiation matrices to sparse matrices. Wei usually chooses the localizer to be a Gaussian, $s(X) \equiv \exp \left(-X^{2} / L^{2}\right)$ for some positive constant $L[4,37,17,1,14,15]$, but our results are general, and only restricted to Gaussian-DSC where explicitly noted. The Gaussian-localized basis was independently invented as the "sinc-Gaussian" interpolation [34]. Whatever the name, some DSC/sinc-Gaussian convergence and error theory can be found in $[34,25,28,30,29,31]$.

Radial basis functions (RBFs) are a popular method for multidimensional interpolation on irregular or scattered grids $[11,38,10,33]$ and for solving differential equations $[13,16,19,20,21,22,26,27,39]$. RBFs seem at first glance to have little connection with the DSC scheme. In any number of dimensions $d$, $\mathrm{RBF}$ basis functions are of the form :

$$
\phi_{j} \equiv \phi\left(\left\|\vec{x}-\vec{x}_{j}\right\|_{2}\right) \quad \vec{x} \in R^{d}
$$

for some univariate function $\phi(r)$ and some set of $N$ points $\vec{x}_{j}$, which are called the "centers". (Many species of $\phi(r)$ have been used in the literature as reviewed in [18]). The error falls exponentially with $N$ for smooth $f(\vec{x})$ and certain choices of $\phi(r)$; these "spectrally accurate" RBFs contain a "shape parameter" or "relative inverse width" $\alpha$. We shall write the various RBF species $\phi$ as $\phi([\alpha / h] x)$. The user-choosable constant $\alpha$ is the "shape parameter" or "relative inverse width" [these terms are synonyms]. The RBF width is written as the ratio $\alpha / h$ because only the width relative to the grid spacing is significant. 
The RBF basis functions can be recombined into cardinal bases, but unfortunately, the localizers equivalent to the standard RBF species $\phi(r)$ are not known in exact explicit form. However, as shown in [7, 24], the cardinal function localizer for Gaussian RBFs is, to very accurate approximation,

$$
s(X)=\frac{\alpha^{2} X}{\sinh \left(\alpha^{2} X\right)}\left\{1+O\left(\exp \left(-2 \pi^{2} / \alpha^{2}\right)\right)\right\} \quad \text { [Gaussian RBF localizer] }
$$

where $\alpha$ is the shape parameter of the RBFs. (In the limit $\alpha \rightarrow 0$, the localizer for Gaussian RBFs becomes $s(X) \approx 1$ over an increasingly large interval in $X$, thus yielding an alternative proof of the theorem by Riemenschneider and Sivakumar [32] that Gaussian RBFs on an unbounded domain tend to a sinc series in the limit $\alpha \rightarrow 0$.)

Later work by the author showed that the localizer for sech, inverse quadratic (IQ), multiquadric (MQ) and inverse multiquadric (IMQ) RBFs is also approximately of the form $s(X)=(\pi X / \rho) / \sinh (\pi X / \rho)$. The localizer width $\rho$ depends on the shape parameter of the RBFs with a different functional form for each RBF species [5] as catalogued in Table 1. The same article shows that the multidimensional Gaussian RBF cardinal function on a uniform square lattice is, without approximation, the product of the corresponding one dimensional cardinal functions. The same tensor product factorization property is true, though only approximately, for multidimensional sech, IQ, MQ and IMQ cardinal functions. Collectively, we shall dub these 5 RBF species the "sinh-localized RBFs".

Because of this similarity of form, the Fourier Transform of all these RBF cardinal functions has the common shape, exponentially decaying for $|K|>\pi$,

$$
\begin{aligned}
\bar{C}(K) & \sim \frac{1}{1+2 \exp (-\pi \rho) \cosh (\rho K)}, \quad K \in[-2 \pi, 2 \pi] \\
& \approx \frac{1}{2}\left\{\tanh \left(\frac{1}{2} \rho(K+\pi)\right)-\tanh \left(\frac{1}{2} \rho(K-\pi)\right)\right\}
\end{aligned}
$$

where the two forms are equivalent with a relative error of $O(\exp (-2 \pi \rho))$ as shown in [5]. This common form for the Fourier transform of the cardinal function will appear in the Fourier error theorem proved below.

Thus, there is great interest in an error theorem for basis functions of localized-sinc form as proved in Sec. 3. It was previously known that when $\alpha$ is large, that is, when the RBFs are very narrow, the approximation error for a smooth $f(x)$ is huge because the smooth function is approximated by a finite sum of narrow spikes. In between the spikes, all the basis functions are near zero and so $f^{R B F}$ must fall to zero even though $f(x)$ does not.

In the opposite limit, RBFs tend to the sinc basis. This was first proved for Gaussian RBFs in [32]. The theorem proved below, together with the approximations to the RBF cardinal function that are exact in the limit $\alpha \rightarrow 0$, is a new proof that the flat limit of RBFs is a sinc approximation for all five species of RBFs considered here. 


\section{A Theorem on Errors in Fourier Transform Space}

Sinc and sinc-localized interpolation is simpler than competitors like Chebyshev interpolation because the sinc and sinc-cardinal functions $C_{j}(x ; h) \equiv s([x-$ $j h] / h) \operatorname{sinc}([x-j h] / h)$ are all translates (by an integer multiple of the grid spacing $h$ ) and dilations (by $h$ ) of a single function, dilated so its roots fall on the unit grid, $C(X)=s(X) \operatorname{sinc}(x)$, which we dub the "master cardinal function".

To prove the error theorem, we first need Fourier Transforms of cardinal functions which are given by the following:

Theorem 1 (localized-Sinc Cardinal Function Fourier Transform) Define $S(K)$ as the Fourier Transform of the localizer function $s(X)$ :

$$
S(K) \equiv \mathcal{F} \mathcal{T}\{s(X)\}(K) \equiv \int_{-\infty}^{\infty} \exp (i K X) s(X) d X
$$

and recall that the cardinal functiions are generated from Define

$$
C(X) \equiv s(X) \operatorname{sinc}(\mathrm{X})
$$

Then the Fourier Transforms of the cardinal functions are

1.

$$
\bar{C}(K) \equiv \mathcal{F} \mathcal{T}\{s(X) \operatorname{sinc}(\mathrm{X})\}(\mathrm{K})=\frac{1}{2 \pi} \int_{\mathrm{K}-\pi}^{\mathrm{K}+\pi} \mathrm{S}(\mathrm{Y}) \mathrm{d} \mathrm{Y}
$$

2.

$$
\mathcal{F} \mathcal{T}\left\{s\left(\frac{x-j h}{h}\right) \operatorname{sinc}\left(\frac{x-j h}{h}\right)\right\}(k)=\mathcal{F} \mathcal{T}\{s(x) \operatorname{sinc}(x)\}(k h) h \exp (i j h k)
$$

Proof: Proposition 1 is an expression for the transform of the cardinal function in terms of the transform of the mollifer $s(X)$. This can be derived through the Convolution Theorem, which asserts that for any pair of functions $s(X)$ and $\operatorname{sinc}(X)$

$$
\mathcal{F} \mathcal{T}\{s(X) \operatorname{sinc}(X)\}(K)=\frac{1}{2 \pi} \int_{-\infty}^{\infty} \mathcal{T}(K-Y, \pi) S(Y) d Y
$$

where we have inserted the Fourier Transform of the sinc function,

$$
\mathcal{F} \mathcal{T}\{\operatorname{sinc}(X)\}(K)=\mathcal{T}(K ; \pi) \equiv \begin{cases}1, & |K| \leq \pi \\ 0, & |K|>\pi\end{cases}
$$


(Note that for the special case $s(x) \equiv 1$, the sinc basis, the transform of the sinc cardinal function is $\bar{C}^{\text {sinc }}(K)=\mathcal{T}(K ; \pi)$.) The Convolution Theorem becomes

$$
\begin{aligned}
& \bar{C}(K) \equiv \mathcal{F} \mathcal{T}\{s(X) \operatorname{sinc}(X)\}(K)=\frac{1}{2 \pi} \int_{-\infty}^{\infty} \mathcal{T}(K-Y ; \pi) S(Y) d Y \\
& =\frac{1}{2 \pi} \int_{-\infty}^{\infty}\left\{\begin{array}{cc}
S(Y), & |K-Y| \leq \pi \\
0, & |K-Y|>\pi
\end{array}\right\} d y \\
& =\frac{1}{2 \pi} \int_{K-\pi}^{K+\pi} S(Y) d Y
\end{aligned}
$$

as claimed.

The second proposition requires an evaluation of the Fourier Transform of the dilated-and-translated master cardinal function

$$
\mathcal{F} \mathcal{T}\{s([x-j h] / h) \operatorname{sinc}([x-j h] / h)\}(k)
$$

However, a standard Fourier Transform identity asserts that for any function $g(x)$ with transform $G(k)$ and any shift $\mathfrak{s}$ (not necessarily an integer) and an arbitrary dilation $h$,

$$
\mathcal{F} \mathcal{T}(g([x-\mathfrak{s}] / h))=\int_{-\infty}^{\infty} g([x-\mathfrak{s}] / h) \exp (i k x) d x=h G(h k) \exp (i \mathfrak{s} k),
$$

as can be proved by a simple change of coordinate in the integral. Applying this with $\mathfrak{s}=j h$ and $g(x)=s(x) \operatorname{sinc}(x)$,

$$
\mathcal{F} \mathcal{T}\{s([x-j h] / h) \operatorname{sinc}([x-j h] / h)\}(k)=\mathcal{F} \mathcal{T}\{s(x) \operatorname{sinc}(x)\}(k h) h \exp (i j(2 k))
$$

Theorem 2 (Localized-Sinc Error Theorem) Define

$$
f^{\text {localized-sinc }}(x ; h) \equiv \sum_{j=-\infty}^{\infty} f(j h) s([x-j h] / h) \operatorname{sinc}([x-j h] / h)
$$

for some localizer function $s(X)$.

Define

$$
F^{\text {folded }}(k) \equiv h \sum_{j=-\infty}^{\infty} f(j h) \exp (i j h k)=\sum_{m=-\infty}^{\infty} F(k-2 \pi m / h)
$$

where the two forms are equivalent under the Poisson Summation Theorem [2]. Denote the error of the RBF or DSC series by

$$
E(x ; h) \equiv f(x)-f^{\text {localized }-\operatorname{sinc}}(x ; h)
$$

Then the following propositions are true: 
1.

$$
\mathcal{F} \mathcal{T}\left\{f^{\text {localized-sinc }}\right\}(k) \equiv F^{\text {folded }}(k ; h) \bar{C}(k h)
$$

2. The Fourier transform $\bar{E}(k)$ of the error $E(x ; h)$ is

$$
\bar{E}(k ; h) \equiv F(k)-F^{\text {folded }}(k ; h) \bar{C}(k h)
$$

Proof:

The first step is to apply the Fourier Transform operator to both sides of the definition of flocalized-sinc,$(22)$.

$\mathcal{F} \mathcal{T}\left\{f^{\text {localized-sinc }}\right\}(k ; h) \equiv \sum_{j=-\infty}^{\infty} f(j h) \mathcal{F} \mathcal{T}\{s([x-j h] / h) \operatorname{sinc}([x-j h] / h)\}(k)$

Next, apply the preceding theorem on Fourier Transforms of cardinal functions, which yields

$$
\begin{aligned}
\mathcal{F} \mathcal{T}\left\{f_{\text {localized-sinc }}\right\}(k ; h) & =\sum_{j=-\infty}^{\infty} f(j h)\{\mathcal{F} \mathcal{T}\{s(x) \operatorname{sinc}(x)\}(k h) \exp (i j h k)\} \\
& =\mathcal{F} \mathcal{T}\{s(x) \operatorname{sinc}(x)\}(k h)\left\{h \sum_{j=-\infty}^{\infty} f(j h) \exp (i j h k)\right\} \\
& =\bar{C}(k h)\left\{h \sum_{j=-\infty}^{\infty} f(j h) \exp (i j h k)\right\}
\end{aligned}
$$

where in the last line we replaced $\mathcal{F} \mathcal{T}\{s(x) \operatorname{sinc}(x)\}(k h)$, the transform of the master cardinal function, by the symbol we defined to be this transform, $\bar{C}$. Substituting the definition of $F^{\text {folded }}$, Eq. (23), for the expression in braces in the last line gives the first proposition (25).

The second proposition for the error then follows immediately by taking the Fourier Transform of $E(X) \equiv f(x)-f^{\text {localized-sinc }}(x ; h)$ and invoking the first proposition.

\section{Error Norm in Physical Space}

In most applications, we are more interested in the error in physical space than in Fourier transform space. It is therefore helpful that the $L_{2}$ error norm in physical space is identical with the $L_{2}$ error norm in Fourier space because of the Parseval identity,

$$
\|E(x)\|_{2}^{2} \equiv \int_{-\infty}^{\infty}|E(x)|^{2} d x=\int_{-\infty}^{\infty}|\bar{E}(k)|^{2} d k \quad[\text { Parseval identity }]
$$




\section{Background for Interpretation}

\subsection{Asymptotics of the Fourier Transform}

A detailed interpretation of the error theorem requires knowledge not only of the transforms of the RBF, DSC and sinc cardinal functions, but also of the transform $F(K)$ of the function which is being approximated. Fortunately, it is possible to make the following general statements:

Theorem 3 (Decay of Fourier Transform) Suppose that $f(x)$ is analytic in the complex $x$-plane in the strip $-\mu-\delta<\Im(x)<\mu+\delta$ where $\mu$ is positive and finite and where the positive constant $\delta$, which slightly widens the required strip of analyticity, may be arbitrarily small and furthermore $f(x)$ satisfies

$$
f(x)=O(\exp (-[q-\epsilon]|\Re(x)|)) \quad \text { as }|x| \rightarrow \infty
$$

for any $\epsilon>0$ and some $q>0$. Then the Fourier Transform exists, is analytic in the strip $-q<\Im(k)<q$ and satisfies

$$
F(k)=O(\exp (-\mu|\Re(k)|)) \quad \text { as }|k| \rightarrow \infty
$$

This is equivalent to the statement that

$$
F(k) \sim A(k) \exp (-\mu|k|) \text { as }|k| \rightarrow \infty
$$

where $A(k)$ is a factor that may oscillate and also contain slower-than-exponential factors such as a combination of powers and logarithms of $k$, but no growing or decaying exponentials.

[Theorem 12.4 on pg. 229 of [40].]

The crucial takeaway is that functions which are analytic on the entire real axis and also decay rapidly - this is precisely the class of $f(x)$ whose sinc, DSC and RBF expansions will converge exponentially fast - have Fourier Transforms which decay exponentially fast, too. We will show that the knowledge that $F(k)$ asymptotically decays proportionally to $\exp (-\mu|k|)$ for some positive constant $\mu$ is sufficient to understand much about sinc, DSC and RBF errors.

\subsection{A Brief Digression on Symmetry}

The RBF basis smears out the step functions (in $k$ ) of sinc theory. This in turn yields dramatically different behavior for symmetric versus antisymmetric $f(x)$ as we shall show below. Because an arbitrary function can always be separated into its symmetric and antisymmetric parts, it is helpful to analyze $f(x)$ that are of even or odd parity with respect to $x=0$, that is, are symmetric or antisymmetric. We can then recover the general case by adding the contributions of the target function's symmetric and antisymmetric parts. It is then further convenient to restrict attention to positive $k$; for $F^{s y m}(k)$ and $F^{\text {anti }}(k)$, the errors for negative $k$ are by symmetry equal in magnitude to those for $k>0$. 
First, note that an arbitrary $f(x)$ can always be decomposed into its symmetric and antisymmetric parts

$$
f(x)=f^{s y m}(x)+f^{a n t i}(x)
$$

where

$$
f^{\text {sym }}(x)=f^{\text {sym }}(-x) \quad \& \quad f^{\text {anti }}(x)=-f^{\text {anti }}(-x)
$$

for all $x$ where

$$
f^{\text {sym }}(x) \equiv(f(x)+f(-x)) / 2 \quad \& \quad f^{\text {anti }}(x) \equiv(f(x)-f(-x)) / 2
$$

Since the Fourier Transform is a linear operator, the symmetric and antisymmetric parts of $f(x)$ transform independently of one another. The Fourier Transform of the symmetric part, $F^{s y m}(k)$, is real-valued if $f^{s y m}(x)$ is real-valued and is always symmetric with respect to $k=0$. Similarly, $F^{\text {anti }}(k)$ is pure imaginary if $f^{a n t i}(x)$ is real, and the transform is antisymmetric with respect to $k=0$.

In the following sections and also in Part 2 [6], we shall find it very illuminating to analyze the symmetric and antisymmetric parts of $f(x)$ separately.

\subsection{Splitting the Error}

If $F(k)$ decays exponentially with $|k|$ as justified above, then the infinite series for $F^{\text {folded }}$ can be simplified to just two terms, and the RBF error becomes

$$
\begin{aligned}
\bar{E}(k) & \approx F(k)-\bar{C}(k h ; \rho(\alpha))\{F(k)+F(k-2 \pi / h)\}, \quad k \in[\delta, 2 \pi / h-\delta] \\
& \approx \bar{E}_{T}+\bar{E}_{D}
\end{aligned}
$$

where

$$
\bar{E}_{T} \equiv F(k)\left\{1-\bar{C}(k h ; \rho(\alpha)\} \quad \& \quad \bar{E}_{D} \equiv-F(k-2 \pi / h) \bar{C}(k h ; \rho(\alpha)\right.
$$

This split of the error is convenient for two reasons. First, for a symmetric $f(x)$, invoking symmetry shows that, with $k \equiv \pi / h+\kappa$,

$$
\begin{aligned}
F^{s y m}(k) & =F^{s y m}(\pi / h+\kappa) \\
F^{s y m}(k-2 \pi / h) & =F^{s y m}(\pi / h-\kappa)
\end{aligned}
$$

We shall see below that the error is concentrated around $|k|=\pi / h$; thus, $F^{\text {sym }}(k-2 \pi / h)$ will have the same sign as $F^{\text {sym }}(k)$ at $k=\pi / h$ and in at least a small neighborhood around it whereas for an antisymmetric function, the signs are opposite, though the magnitudes of $F^{a n t i}(k)$ and $F^{a n t i}(k-2 \pi / h)$ are still the same. The two parts of the error will therefore constructively add or destructively partially cancel, depending on the symmetry of $f(x)$.

The second reason for the error splitting is that these two error terms have simple but different interpretations in the sinc limit, $\alpha \rightarrow 0$. The term $\bar{E}_{T}$ is identically zero for wave numbers $|k| \geq \pi / h \equiv k_{\text {alias }}$ and is therefore the "truncation error" due to the omission of all wave numbers beyond the aliasing 
limit in the sinc approximation. The term $E_{D}$ is the "discretization error" or "aliasing error"; higher wave numbers are spuriously aliased to wave numbers on the interval $k \in\left[-k_{\text {alias }}, k_{\text {alias }}\right]$. With an RBF or DSC basis, both errors are diffused as explained above, but the distinction between these two contributions to total error remains conceptually useful.

\section{$5 \quad$ Sinc Pseudospectral Errors}

To understand how mollification has changed the interpolation error, it is useful to look at the special case of unlocalized interpolation, i. e., classic sinc interpolation.

The Fourier transform of the error in the sinc basis is

$$
\begin{aligned}
\bar{E}^{\text {sinc }}(k ; h) & =F(k)-\mathcal{T}(k h ; \pi) F^{\text {folded }}(k ; h) \\
& = \begin{cases}-\sum_{m=-\infty, m \neq 0}^{\infty} F(k h-2 \pi m) & |k| \leq \pi / h \\
F(k), & |k|>\pi / h\end{cases}
\end{aligned}
$$

where $\mathcal{T}(k h ; \pi)$ is the top-hat function, equal to one for $|k| \leq \pi / h$ and zero elsewhere. (Recall that $\bar{C}^{\operatorname{sinc}}(K)=\mathcal{T}(K ; \pi)$.)

The sinc approximation is "bandlimited" in the sense that $\mathcal{F} \mathcal{T}\left(f^{\text {localized }-s i n c}(k ; h)\right.$ is zero for all $|k|>\pi / h$. The Shannon-Kotelnikov-Whittaker Sampling Theorem [12] states that if $f(x)$ is bandlimited, that is, if $F(k)=0$ for all $|k| \geq \pi / h$, the sinc approximation with grid spacing $h$ is exact. These facts, long known in signal processing, imply that all the error for a non-bandlimited function must come entirely from $F(k)$ for $|k|>\pi / h$.

The crucial wavenumber $k=\pi / h$ is usually called the "aliasing wavenumber"

$$
k_{\text {alias }} \equiv \pi / h
$$

The reason for the name is that $\exp \left(i k_{\text {alias }} x\right)$ is a wave with a wavelength of $2 h$, which is the shortest oscillation that can be represented on a uniform grid of spacing $h$. All waves with $|k|>k_{\text {alias }}$ will be aliased to shorter waves as explained in [3].

The aliasing of each wavenumber $k$ such that $|k|>k_{\text {alias }}$ triggers two errors simultaneously. First, there is a "truncation error"; because the sinc approximation is bandlimited, all the high wavenumbers such that $|k|>\pi / h$ are zero in the approximation:

$$
\bar{E}_{T}^{\text {sinc }}(k ; h)= \begin{cases}0, & |k| \leq \pi / h \\ F(k), & |k|>\pi / h\end{cases}
$$

The second error, dubbed the "discretization error" in [3] and in the previous section, arises because aliasing distorts the Fourier spectrum of $F^{\operatorname{sinc}}(k)$ for 
$|k|<\pi / h$. This discretization error is

$$
\begin{aligned}
\bar{E}_{D}^{\text {sinc }}(k ; h) & =\left\{\begin{array}{l}
F(k)-F^{\text {folded }}(k ; h), \quad|k| \leq \pi / h \\
0, \quad|k|>\pi / h
\end{array}\right. \\
& =-F(k h-2 \pi)-F(k h+2 \pi)-\sum_{m=-\infty, m \neq 0, \pm 1} F(k h-2 \pi m)
\end{aligned}
$$

where the last line is restricted to $|k| \leq \pi / h$. If $F(k)$ is decaying exponentially fast with $|k|$ and $h$ is small enough so that $F(k)$ is very small for $|k|>\pi / h$, the aliasing limit, then the summation in the second line of (42) can be neglected. Indeed, for positive $k, F(k h+2 \pi)$ is negligible, too, leaving only, for small but otherwise arbitrary $\delta>0$ :

$$
F^{\text {folded }}(k h) \approx F(k h)+F(k h-2 \pi), \quad k \in[\delta / h, 2 \pi / h-\delta / h] .
$$

The sinc error is

$$
\begin{aligned}
\bar{E}^{\text {sinc }}(k h) & =F(k h)-\mathcal{T}(k h ; \pi) F^{\text {folded }}(k h) \\
& \approx F(k h)-\mathcal{T}(k h ; \pi)\{F(k h)+F(k h-2 \pi)\} \\
& \approx \begin{cases}-F(k h-2 \pi), \quad k \in[\delta / h, \pi / h] \\
F(k h), \quad k \in[\pi / h, 2 \pi / h-\delta / h]\end{cases}
\end{aligned}
$$

(Note that at $k=0$, the sinc error is approximately $F(-2 \pi)+F(2 \pi)$; the restriction to $k>\delta / h$ excludes the region where $F(k h+2 \pi)$ makes a significant contribution to $F^{\text {folded }}(k ; h)$.)

If we assume that $F(k)$, the Fourier Transform of $f(x)$, decays exponentially as $|k| \rightarrow \infty$, then Fig. 1 is a schematic of the errors. The crucial point is that these errors (in Fourier space) are dominated by errors near the "aliasing wavenumber", $k_{\text {alias }}=\pi / h$, as claimed earlier. The error decays exponentially with $k$ in either direction away from these peaks.

\section{Diffusion in Fourier Wavenumber Space}

All DSC and sinh-localized RBF errors have the form in Fourier space of

$$
\bar{E}(k)=F(k)-\bar{C}(k h) F^{\text {folded }}(k ; h)
$$

In this formula, the sole difference between different basis sets is the difference in $\bar{C}(k h)$, the Fourier Transform of the cardinal function on the unit grid. For the sinc basis, the transform of the cardinal function is the "top-hat" function, also known as a "rectangle" function or "normalized boxcar" function, that is one on the interval $k \in[-\pi / h, \pi / h]$ and zero for all other $k$, i. e.,

$$
\bar{C}^{\text {sinc }}(k h)=\mathcal{T}(k ; \pi / h)=\left\{\begin{array}{l}
1, k \in[-\pi / h, \pi / h] \\
0,|k|>\pi / h
\end{array}\right.
$$

The DSC and RBF cardinal functions are smoothed top-hat functions. 
To define precisely what we mean by "smoothed", recall that the diffusion equation,

$$
u_{t}=u_{x x}, \quad u(x, 0)=q(x), \quad x \in[-\infty, \infty]
$$

is solved by the convolution integral

$$
u(x, t)=\int_{-\infty}^{\infty} \frac{1}{2 \sqrt{\pi t}} \exp \left(-\frac{(x-y)^{2}}{4 t}\right) q(y) d y
$$

If the initial condition is a top-hat function $\mathcal{T}(k ; \pi)$, then $u(x, t)$ for any positive $t$ is the sum of two error functions; this sum is also the Fourier Transform of the DSC cardinal function. In other words, the DSC is the result of diffusively smoothing the top-hat transform of the sinc cardinal function in Fourier space.

For the sinh-localized RBFs, the smoothing is a different convolution:

$$
u(x, t)=\int_{-\infty}^{\infty} \frac{\pi}{4 t} \operatorname{sech}^{2}\left(\frac{\pi}{2 t}(x-y)\right) q(y) d y
$$

where "time" $t=\pi /(\rho h)$. If $q=\mathcal{T}(x ; \pi)$, then $u$ is the shape of the Fourier Transform $\bar{C}$ of the RBF cardinal function. Although (51) is not the solution to any simple partial differential equation, it is similar to diffusion in that it is also a convolution with a kernel that is symmetric and monotonically falls in either direction from its peak.

If the smoothing is weak, then the approximation will be close to the sinc approximation and therefore spectrally-accurate. This provides an intuitive justification for RBF and DSC methods.

\section{Analysis of the Consequences of Mollifying the Sinc Basis}

Is this smearing of the sinc error good? The answer is: It depends.

Sinc interpolation employs a "guillotine" truncation in Fourier space. That is to say, all wave numbers less than the aliasing limit are captured perfectly. All wave numbers $k$ larger than the aliasing wave number $\pi / h$ are aliased to lower wave numbers and thus completely misrepresented. There is no smooth transition from fidelity to failure: the transition is as abrupt as a slice of the guillotine blade.

In reality, the Fourier transform $F(k)$ does not stop abruptly at some wave number, but rather smoothly and exponentially decays are larger and larger wave number all the way to infinity. The Fourier space diffusion means that the same smooth decay is likewise a property of the error of sinc-localized interpolation. If $f(x)$ is symmetric in $x, F(k)$ will be symmetric in wave number. It is then possible by tuning the degree of mollification by a wise choice of the RBF shape parameter $\alpha$ to match this beyond-the-aliasing-limit behavior of $F(k)$ in its sinc-localized approximation. This makes it possible for RBFs to sometimes be much more accurate than sinc interpolation - "spectral-plus". This is true even though RBFs tends to sinc interpolation in the "flat limit" that $\alpha \rightarrow 0$. 
Alas, this happy situation that RBFs are "spectral-plus" is rather special. It requires that $f(x)$ be symmetric. It also requires careful tuning of the RBF shape parameter. Doing justice to the relative merits of localized versus unlocalized sinc interpolation demands an extensive study with lots of examples as provided in Part 2 [6]. 


\section{Summary}

On a uniform, unbounded grid, sinh-localized RBFs and the Discrete Singular Convolution (DSC) basis of Wei are here provided with a simple error analysis in Fourier space. If a function $f(x)$ is such that its Fourier Transform decays exponentially fast with wavenumber, then we show that the sinc, RBF and DSC methods will converge exponentially fast, too.

All five RBF "shift-invariant" RBF species (Gaussian, sech, Multiquadric, Inverse Multiquadric and Inverse Quadratic) converge to the sinc basis as the RBF shape parameter $\alpha \rightarrow 0$; these five basis sets are essentially the same [5]. This was previously known, but our new theorem provides a novel proof of the "flat limit" behavior of these RBFs.

We have shown that mollification effectively diffuses the error of unqualified sinc interpolation, but the diffusion is not in physical space but rather in the space of Fourier wave numbers $k$. We show that this diffusion makes it possible for radial basis functions to sometimes be much more accurate than sinc interpolation, but only under special conditions. The full treatment of the relative merits of localized versus unlocalized sinc interpolation requires much further analysis and many examples and is therefore postponed to a second article [6].

The most interesting applications of radial basis functions are not to a onedimensional, uniform grid, but rather to an irregular grid in two or more dimensions. Nevertheless, this paper and its companions $[8,7,9,6]$ have given some useful insights for practical applications.

This work was supported by NSF grants OCE 0451951, ATM 0620100 and ATM 0723440.

\section{References}

[1] G. Bao, G. W. Wei, And S. Zhao, Numerical solution of the Helmholtz equation with high wavenumbers, Inter. J. Numer. Meths. Engrg., 59 (2004), pp. 389-408.

[2] J. P. Boyd, New directions in solitons and nonlinear periodic waves: Polycnoidal waves, imbricated solitons, weakly non-local solitary waves and numerical boundary value algorithms, in Advances in Applied Mechanics, T.Y. Wu and J. W. Hutchinson, eds., no. 27 in Advances in Applied Mechanics, Academic Press, New York, 1989, pp. 1-82.

[3] - Chebyshev and Fourier Spectral Methods, Dover, Mineola, New York, 2d ed., 2001. 665 pp.

[4] — A proof that the discrete singular convolution (DSC)/LagrangeDistributed Approximating Function (LDAF) method is inferior to high order finite differences, J. Comput. Phys., 214 (2006), pp. 538-549.

[5] — The near-equivalence of five species of spectrally-accurate radial basis functions (RBFs): Asymptotic approximations to the RBF cardinal 
functions on a uniform, unbounded grid, J. Comput. Phys., 230 (2011), pp. 1304-1318.

[6] - A Fourier error analysis for radial basis functions and the Discrete Singular Convolution on an infinite uniform grid, Part 2: Spectral-Plus is special, J. Sci. Comput., (2013). to be submitted.

[7] J. P. BOYD AND L. WANG, An analytic approximation to the cardinal functions of Gaussian radial basis functions on a one-dimensional infinite uniform lattice, Appl. Math. Comput., 215 (2009), pp. 2215-2223.

[8] _ Truncated Gaussian RBF differences are always inferior to finite differences of the same stencil width, Commun. Comput. Phys., 5 (2009), pp. $42-60$.

[9] — Asymptotic coefficients for Gaussian radial basis function interpolants, Appl. Math. Comput., 216 (2010), pp. 2394-2407.

[10] M. D. Buhmann, Radial basis functions, Acta Numerica, 9 (2000), pp. 138.

[11] — Radial Basis Functions: Theory and Implementations, vol. 12 of Cambridge monographs on Applied and Computational Mathematics, Cambridge University Press, 2003.

[12] P. L. Butzer, P. J. S. G. Ferreira, J. R. Higgins, S. Saitoh, G. Schmeisser, And R. L. Stens, Interpolation and sampling: E.T. Whittaker, K. Ogura and their followers, J. Fourier Anal. Applic., 17 (2011), pp. 320-354.

[13] P. P. Chinchapatnam, K. Djidjeli, and P. B. Nair, Unsymmetric and symmetric meshless schemes for the unsteady convection-diffusion equation, Comput. Meths. Appl. Mech. Engrg., 195 (2006), pp. 2432-2453.

[14] O. CivaleK, Discrete singular convolution method and applications to free vibration analysis of circular and annular plates, Structural Engrg. Mech., 29 (2008), pp. 237-240.

[15] _ - Vibration analysis of conical panels using the method of discrete singular convolution, Commun. Numer. Meths. Engrg., 24 (2008), pp. 169181.

[16] A. I. Fedoseyev, M. J. Friedman, and E. J. Kansa, Improved multiquadric method for elliptic partial differential equations via PDE collocation on the boundary, Comput. Math., 43 (2002), pp. 439-455.

[17] B. F. Feng And G. W. WeI, A comparison of the spectral and the discrete singular convolution schemes for the KdV-type equations, J. Comput. Appl. Math., 145 (2002), pp. 183-188. 
[18] B. Fornberg And N. Flyer, Accuracy of radial basis function interpolation and derivative approximations on 1-D infinite grids, Adv. Comput. Math., 23 (2005), pp. 5-20.

[19] E. J. KANSA, Multiquadrics-a scattered data approximation scheme with applications to computational fluid dynamics-II. Solutions to hyperbolic, parabolic, and elliptic partial differential equations, Comput. Math. Appl ., 19 (1990), pp. 147-161.

[20] E. J. Kansa, H. Power, G. E. Fasshauer, And L. Ling, A volumetric integral radial basis function method for time-dependent partial differential equations: I. Formulation, J. Engrg. Anal. Boundary Elements, 28 (2004), pp. 1191-1206.

[21] Q. T. LE GIA, Approximation of parabolic PDEs on spheres using spherical basis functions, Adv. Comput. Math., 22 (2005), pp. 377-397.

[22] L. Ling, R. Opfer, And R. Schaback, Results on meshless collocation techniques, Engrg. Anal. Boundary Elements, 30 (2006), pp. 247-253.

[23] J. Lund And K. L. Bowers, Sinc Methods For Quadrature and Differential Equations, Society for Industrial and Applied Mathematics, Philadelphia, 1992. $304 \mathrm{pp}$.

[24] V. Maz'ya and G. Schmidt, Approximate Approximations, American Mathematical Society, Providence, 2007. 349 pp.

[25] C. A. Micchelli, Y. Xu, And H. Zhang, Optimal learning of bandlimited functions from localized sampling, J. Complexity, 25 (2009), pp. 85-114.

[26] T. J. Moroney and I. W. Turner, A three-dimensional finite volume method based on radial basis functions for the accurate computational modeling of nonlinear diffusion equations, J. Comput. Phys., 225 (2007), pp. 1409-1426.

[27] R. B. Platte and T. A. Driscoll, Computing eigenmodes of elliptic operators using radial basis functions, Comput. Math. Appl., 48 (2006), pp. $1251-1268$.

[28] L. QIAN, On the regularized Whittaker-Kotel'nikov-Shannon sampling formula, Proc. Amer. Math. Soc., 131 (2003), pp. 1169-1176.

[29] L. W. QIAN AND D. B. CREAmer, Localization of the generalized sampling series and its numerical application, SIAM J. Numer. Anal., 43 (2006), pp. 2500-2516.

[30] — - A modification of the sampling series with a Gaussian multiplier, Sampling Theory in Signal and Image Processing, 5 (2006), pp. 307-325. 
[31] L. W. QIan And H. OgaWA, Modified sinc kernels for the localized sampling series, Sampling Theory in Signal and Image Processing, 4 (2005), pp. 121-139.

[32] S. D. Riemenschneider And N. Sivakumar, On cardinal interpolation by Gaussian radial-basis functions: Properties of fundamental functions and estimates for Lebesgue constants, J. Analyse Math., 79 (1999), pp. 3361 .

[33] R. Schaback and H. Wendland, Kernel techniques: From machine learning to meshless methods, Acta Numerica, 15 (2006), pp. 543-639.

[34] G. Schmeisser And F. Stenger, Sinc approximation with a Gaussian multiplier, Sampling Theory in Signal and Image Processing, 6 (2007), pp. 199-221.

[35] F. Stenger, Sinc Methods, Springer-Verlag, New York, 1993. 500 pp.

[36] F. Stenger, Summary of sinc numerical methods, J. Comput. Appl. Math., 121 (2000), pp. 379-420.

[37] G. W. Wei and S. Zhao, On the validity of 'a proof that the discrete singular convolution $(D S C) /$ Lagrange-distributed approximation function (LDAF) method is inferior to high order finite differences', J. Comput. Phys., 226 (2007), pp. 2389-2392.

[38] H. Wendland, Scattered Data Approximation, Cambridge University Press, 2005.

[39] S. M. Wong, Y. C. Hon, And M. A. Golberg, Compactly supported radial basis functions for shallow water equations, Appl. Math. Comput., 127 (2002), pp. 79-101.

[40] A. I. ZAYED, Handbook of Function and Generalized Function Transformations, vol. 3 of Mathematical Sciences Reference Series, CRC Press, Boca Raton, FL, 1996.

Table 1: $\rho$ in $C(X) \sim(1 /(\rho)) \sin (\pi X) / \sinh (\pi X / \rho)$

\begin{tabular}{|c|c|c|}
\hline Type & $\phi$ [unit grid] & $\rho(\alpha)$ \\
\hline Gaussian & $\exp \left(-\alpha^{2} X^{2}\right)$ & $\pi / \alpha^{2}$ \\
sech & $\operatorname{sech}(\alpha X)$ & $\pi / \alpha$ \\
IQ & $1 /\left(1+\alpha^{2} X^{2}\right)$ & $2 / \alpha$ \\
MQ & $\sqrt{1+\alpha^{2} X^{2}}$ & $(2 / \alpha)\left(K_{0}(\pi / \alpha) / K_{1}(\pi / \alpha)\right)+(4 / \pi)$ \\
IMQ & $1 / \sqrt{1+\alpha^{2} X^{2}}$ & $\left(2 K_{1}(\pi / \alpha) /\left(\alpha K_{0}(\pi / \alpha)\right)\right.$ \\
\hline
\end{tabular}




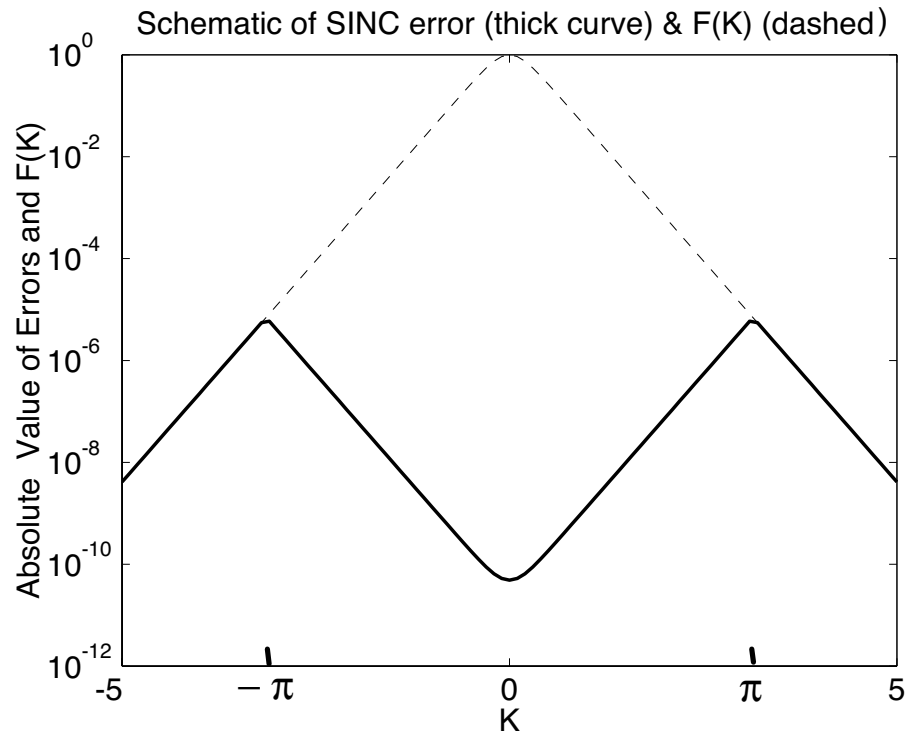

Figure 1: Absolute values of $F(k)$, the Fourier Transform of $f(x)$, (thin dotted curve) and of the total error $\bar{E}^{s i n c}(k ; h)$ when approximating $f(x)$ by a sinc series (thick solid curve). The schematic assumes a typical $f(x)$ that is analytic on a strip of some width $\mu>0$ about the whole real axis and decays rapidly as $|x| \rightarrow \infty$. This implies that $F(k)$ is exponentially-decaying proportionally to $\exp (-\mu|k|)$ for large $k$; this rate of decay appears linear on this log-linear graph. The horizontal axis is $K \equiv k h$ where $h$ is the grid spacing in $x$. The peaks of the error at $K= \pm \pi$, equivalent to $k= \pm k_{\text {alias }}= \pm \pi / h$. 